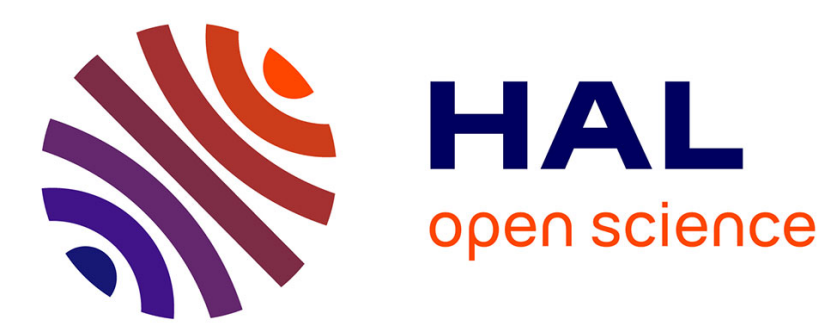

\title{
Paradigme environnemental et action publique urbaine à Monterrey (Mexique) \\ Patrice Mele
}

\section{To cite this version:}

Patrice Mele. Paradigme environnemental et action publique urbaine à Monterrey (Mexique). Autrepart - Revue de sciences sociales au Sud, 2002, 21, pp.41-54. 10.3917/autr.021.0041 . halshs00005672

\section{HAL Id: halshs-00005672 \\ https://shs.hal.science/halshs-00005672}

Submitted on 14 Nov 2010

HAL is a multi-disciplinary open access archive for the deposit and dissemination of scientific research documents, whether they are published or not. The documents may come from teaching and research institutions in France or abroad, or from public or private research centers.
L'archive ouverte pluridisciplinaire HAL, est destinée au dépôt et à la diffusion de documents scientifiques de niveau recherche, publiés ou non, émanant des établissements d'enseignement et de recherche français ou étrangers, des laboratoires publics ou privés. 


\title{
Paradigme environnemental et action publique urbaine à Monterrey (Mexique)
}

\author{
Patrice Melé*
}

Les références à la protection de l'environnement n'ont pas seulement pour utilité de réintroduire la nature et sa protection au sein des débats sur la ville ou de renforcer les actions techniques de contrôle des pollutions. Le paradigme environnemental propose un cadre systémique pour penser la ville de demain [Micoud et alii, 1996] qui implique une action de réorganisation des modalités de production d'espace urbain.

Plusieurs tentatives de comparaisons internationales ont souligné l'intégration des préoccupations environnementales au sein des processus de contrôle de l'urbanisation [Fernandes, 1998] et le rôle d'un modèle de développement urbain durable basé sur la promotion d'une ville compacte, organisée autour d'une utilisation plus intensive du sol, gérée à partir d'une meilleure intégration entre les politiques de transports en commun et la planification de la croissance urbaine [Camagni, Gibelli, 1997]. De plus, les références à l'environnement autorisent une réflexion sur la durabilité des modes de production de l'espace urbain, sur la mise en œuvre de politiques de gestion à l'échelle des aires métropolitaines et sur la réduction des inégalités de conditions de vie [Atkinson, Davila, 1999].

L'intervention des organismes internationaux et des ONG, mais aussi les multiples rencontres entre acteurs politiques et techniques, experts, écologistes et chercheurs ont instauré, à partir de la notion d'environnement, une nouvelle forme d'intérêt général conditionnant une intervention publique spécifique. L'institution conjointe de l'environnement comme " problème » et nouveau champ pour l'action publique [Lascoumes, 1998] a été réalisée au sein d'instances et à partir d'événements internationaux, et présentée comme une tentative de réponse globale à des questions «planétaires».

Le paradigme environnemental est accompagné de la diffusion d'une série de principes d'action et de «bonnes pratiques ». Le principe du pollueur-payeur, le droit de l'information, la participation de la population, la création de comités de concertation entre usagers, la décentralisation, la privatisation de certains services [Marvin, Guy, 1999] et l'éducation environnementale ont été élevés par la doxa environnementale au rang de modèles d'action publique légitime applicables dans

* Centre de recherche «Ville, Société, Territoire », université de Tours. 
tous les contextes. L'intégration de ces principes au sein des législations nationales, la diffusion de ces pratiques au niveau local [Rudolf, 1998] sont dotées de la capacité d'améliorer la qualité de la vie des populations et de garantir la prise en compte des responsabilités à l'échelle planétaire [Micoud et alii, 1996]. Des références à des dynamiques globales justifient la mise en œuvre de «bonnes pratiques de gouvernement local » [Farthing, 1997].

Si la lutte contre les nuisances, pollutions et risques constitue le cœur de l'action publique environnementale, cet article interroge un autre domaine: celui des interactions entre le renforcement de la protection de l'environnement et le contrôle de l'expansion urbaine. Après avoir analysé la construction d'une action publique environnementale, soumise à une forte exposition internationale et instituée dans une tension entre pouvoirs locaux et administrations fédérales, la présentation de situations de conflits et de processus de contrôle de l'urbanisation permettra de préciser le rôle des principaux acteurs impliqués dans l'intégration de référentiels environnementaux au sein de l'action publique urbaine.

Pour l'analyse des mutations de l'action publique urbaine et environnementale, la ville de Monterrey présente plusieurs spécificités intéressantes ${ }^{1}$. Monterrey est une métropole de 3,2 millions d'habitants (en 2000) formée par une aire métropolitaine de neuf municipes gérés en majorité par le Parti d'action national, qui contrôle aussi depuis 1997 le gouvernement de l'État du Nuevo León. Il s'agit, après la ville de Mexico, de la seconde métropole industrielle du Mexique, situation qui place les questions de pollution, de risques et de gestion des interactions entre espaces résidentiels et activités industrielles au centre des débats et des controverses entre habitants et pouvoirs publics. De plus, le site de la ville est entouré de montagnes, protégées par des qualifications juridiques fédérales mais soumises à de fortes pressions de l'urbanisation. Plusieurs conflits urbains pour la protection de la « nature » ont mobilisé des habitants contre la poursuite de l'urbanisation sur ces espaces.

\section{L'institution de l'environnement: diffusion internationale et initiatives locales}

Les modalités de l'action publique environnementale ont été construites au Mexique sous l'impact d'injonctions et de références internationales. Non seulement les conférences de Stockholm (1972) et de Rio (1992) ont exercé, ici comme ailleurs, une forte influence sur la façon de penser l'action publique environnementale, mais l'ouverture économique et la perspective de la signature d'un accord de libre-échange avec le Canada et les États-Unis ont hâté la mise en place de réformes juridiques et institutionnelles. Les réformes libérales des présidents Miguel de la Madrid (1982-1988) et Carlos Salinas de Gortari (1988-1994) prennent la forme d'une libéralisation économique et d'une réduction de l'intervention des

1 La recherche sur la ville de Monterrey qui a été réalisée dans le cadre du projet Medio, projet de recherche franco-mexicain construit à partir d'études de cas dans cinq villes mexicaines (1998-2000) [Melé, Bassols, 2000], repose sur une série d'entretiens avec des responsables locaux de l'aménagement urbain, de l'action publique environnementale et des associations d'habitants et des organisations " écologistes ", entretiens réalisés par l'auteur avec la collaboration d'Adriana Muela. 
pouvoirs publics permettant l'intégration d'un bloc commercial nord-américain tout en affichant la nécessité d'un renforcement des politiques de protection de l'environnement.

L'administration américaine a exercé une forte pression pour une évolution des pratiques mexicaines. La crainte, exprimée par les écologistes et des syndicats américains, d'une délocalisation industrielle massive, rendue possible par les bas salaires et des réglementations environnementales moins draconiennes, a été relayée du côté mexicain par des associations de protection de la nature qui militaient pour le renforcement de la protection des ressources naturelles et soulignaient le risque de l'accueil d'industries polluantes et de déchets toxiques. En outre, face aux difficultés d'accès aux services urbains (eau, drainage, ramassage des ordures ménagère) d'une grande partie des populations urbaines et à la prise de conscience de l'ampleur des risques « urbains » (tremblement de terre de 1985, explosions à Mexico et Guadalajara, inondations, glissements de terrain) et des dangers de la pollution atmosphérique, la qualité de l'environnement urbain est devenue un thème fort pour les opinions publiques et les groupes politiques mexicains.

La loi fédérale de l'équilibre écologique et de protection de l'environnement de 1988 a été considérée par des experts internationaux mais aussi par certains groupes écologiques comme pionnière en Amérique latine [Kurzinger, 1991]. Toutefois son impact est resté limité à cause de la faiblesse des institutions chargées de sa mise en œuvre. La protection de l'environnement se structure en 1992 autour d'institutions renforcées qui restent néanmoins dépendantes d'un grand ministère du Développement social, qui disposait aussi des compétences d'aménagement urbain (Sedesol). Le ministère de l'Environnement est créé en 1994 après l'arrivée au pouvoir d'Ernesto Zedillo (1994-2000), dernier président du Parti révolutionnaire institutionnel. La première ministre de l'Environnement, Julia Carabias, environnementaliste renommée, n'est pas membre du parti officiel et incarne une volonté d'ouverture et de consensus.

Dans le cadre de l'Alena ${ }^{2}$, la protection de l'environnement constitue un thème de «coopération renforcée » entre le Mexique, les États-Unis et le Canada. Une commission de coopération environnementale créée par un accord spécifique est chargée de rendre possible une meilleure coordination des législations et des pratiques. Elle constitue en particulier une incitation à l'évaluation et au renforcement de l'effectivité des législations de chaque État membre.

Mais l'accord de libre-échange nord-américain n'est pas seulement destiné à renforcer la validité et l'effectivité de l'ordre juridique environnemental. Les dispositions de l'Alena protégeant les investissements privés peuvent permettre de considérer certaines contraintes imposées à des entreprises comme "équivalentes à une expropriation ». Le Mexique a été condamné par une instance de régulation des conflits sur la base du non-respect d'une autorisation fédérale d'implantation d'une installation de traitement de déchets toxiques face au refus des autorités locales de délivrer les autorisations nécessaires à sa construction ${ }^{3}$. La possibilité de

\footnotetext{
2 Accord de libre-échange nord-américain.

3 Résolution de la commission d'arbitrage du 30 août 2000 en faveur de l'entreprise Metalcald qui souhaitait s'implanter dans l'État de San Luis Potosi.
} 
ce type de recours place l'ensemble de l'action publique environnementale mexicaine - comme celle des États-Unis et du Canada - sous la menace d'une remise en cause par les règles « juridiques » organisant la libre circulation des capitaux et la protection des investissements internationalement mobiles.

Le renforcement de l'action publique environnementale s'effectue au Mexique dans une tension entre l'action publique fédérale et les initiatives des autorités locales. Selon le fonctionnement particulier du fédéralisme mexicain, les évolutions de la législation fédérale doivent être intégrées au sein des législations des États. Or les relations entre les politiques et les productions juridiques des différents niveaux sont complexes et souvent éloignées des cadres constitutionnels. De nombreuses législations locales n'ont toujours pas instauré localement les innovations des réglementations fédérales. La commission de coopération environnementale comme, sur certains domaines, la Banque mondiale sont particulièrement vigilantes sur les évolutions des législations locales.

La nouvelle législation fédérale de 1996 réformant la loi de 1988 octroie de plus grandes compétences aux autorités locales. Cette répartition des tâches a d'abord nécessité un accroissement des capacités fédérales à légiférer dans ce domaine, réduisant les capacités d'innovations juridiques des États fédérés.

La question des interactions entre action publique environnementale et action publique urbaine est compliquée par la décentralisation de l'action publique urbaine. Alors que les municipalités disposent théoriquement depuis 1983 des compétences d'urbanisme et d'aménagement urbain, certaines législations locales n'ont toujours pas relayé ces dispositions. Les gouvernements des États fédérés ont conservé de fait une grande capacité d'action, en particulier en ce qui concerne l'aménagement urbain des villes capitales d'État.

Le cadre juridique et institutionnel comme l'effectivité de l'action publique urbaine et environnementale peuvent donc être très différents d'une ville à l'autre. Les contextes d'action reflètent non seulement les situations politiques locales, mais aussi le poids au sein des différentes sociétés locales d'acteurs - représentants du secteur privé industriel ou immobilier, propriétaires fonciers - hostiles à la mise en œuvre de processus de contrôle de l'urbanisation. Au Mexique, le cadre d'action défini au niveau fédéral ne s'impose pas aux situations locales. La légitimité comme les conditions d'existence de l'action publique urbaine et environnementale doivent être négociées localement.

\section{Construction de l'action publique environnementale à Monterrey}

Après la publication en 1988 de la loi fédérale de protection de l'environnement, l'intégration des principes de l'action publique environnementale au sein des législations locales de l'État de Nuevo León a été rapide. Une loi de protection de l'environnement de l'État fut promulguée en 1989, et, dès avant 1996, date des réformes de la loi fédérale marquant une généralisation de l'action publique environnementale, 49 des 51 municipalités de l'État avaient publié des règlements d'écologie.

Avec l'appui du président Salinas de Gortari (1988-1994), le gouverneur Socrates Rizzo (1991-1996) a tenté de faire du Nuevo León l'État modèle de la libéralisation et de la modernisation de l'action publique. La construction d'une 
action publique environnementale, dans la ville des principaux groupes industriels du pays, a occupé une place centrale dans cette stratégie. À partir de 1992, ce gouverneur a impulsé un "Pacte écologique du Nuevo León ", à la fois programme d'action et stratégie de communication, pour mobiliser les industriels locaux et diffuser la bonne volonté environnementale du gouvernement de l'État. Un plan environnemental de l'État (1995-2020) proposa un cadre d'action pour la mise en œuvre de mesures de protection de l'environnement.

Dans les projets du gouvernement de l'État est formellement présente, dès avant 1996 et la publication des réformes de la loi fédérale, la construction d'un système autonome de plans écologiques. Le gouvernement de l'État s'était en particulier engagé à intégrer rapidement à la législation locale toute réforme de l'ordre juridique fédéral. Mais cinq ans après les réformes fédérales de 1996, les difficultés pour modifier la loi de protection environnementale de l'État traduisent le changement de la place accordée à l'action publique environnementale.

Après l'éviction de Socrates Rizzo en 1996 avant la fin de son mandat et à la suite de l'élection en 1997 de Fernando Canales Clariond, chef d'entreprise ${ }^{4}$ membre du Parti d'action national, le contexte politique local a été totalement modifié. Les grands entrepreneurs locaux, qui ont abandonné leur stratégie d'alliance avec le PRI saliniste, sont placés directement en position de gestion. On retrouve une évolution similaire au niveau municipal. En 1988, Monterrey constituait une des bases du pouvoir saliniste et le PAN ne gérait que deux des neuf municipes de l'aire métropolitaine. Or, le PRI perd la municipalité de Monterrey dès 1994 et, à partir des élections de 1997, le rapport de forces local est inversé: le PRI ne contrôle plus que deux municipes.

Au niveau fédéral, la protection de l'environnement constitue une priorité réaffirmée d'Ernesto Zedillo, dernier président du PRI. La présence locale des autorités fédérales environnementales est renforcée. Pour le nouveau gouverneur du PAN, en dehors de situations de conflits et de crises liées à des pollutions ou accidents industriels, une certaine distance avec le discours environnemental de ses prédécesseurs et du gouvernement fédéral semble de rigueur.

Par ailleurs, pour la nouvelle administration, qui affiche des convictions libérales et revendique l'application du modèle entrepreneurial aux politiques publiques, l'action publique environnementale est aussi un symbole du renforcement des contrôles administratifs sur les « initiatives privées ». Malgré l'importance des aides fédérales qui ont permis la restructuration et l'internationalisation des groupes industriels de Monterrey, leurs représentants maintiennent une position de principe contre toute intervention de la fédération dans l'économie et toute tentative de planification.

À Monterrey, toute réforme des modalités de l'action publique doit fonder sa légitimité sur une libéralisation, un accroissement de la marge de manœuvre des acteurs privés, les réglementations locales d'urbanisme et les actions environnementales sont soumises à une forte pression des représentants du secteur immobilier liés à la bourgeoisie industrielle.

4 Ex-directeur du groupe des Industries métalliques de Monterrey (IMMSA). 


\section{Acteurs locaux et protection de l'environnement urbain}

Les représentants locaux des autorités environnementales fédérales concentrent leurs activités sur le contrôle de la pollution et des risques industriels ${ }^{5}$, sur la lutte contre le déboisement et la protection des espaces naturels. À Monterrey, un objet juridique improbable a néanmoins (ré)introduit les autorités fédérales dans les débats sur le contrôle de l'urbanisation. Une partie de la croissance de la ville a pris place sur un espace protégé: le Parc national naturel des cimes de Monterrey. En fait, il s'agit d'une protection des montagnes proches de la ville instituée en 1939 par un décret présidentiel de Lazaro Cardenas ${ }^{6}$. La ville implantée à 530 mètres d'altitude est entourée de reliefs culminant entre 2000 et 2500 mètres. Toute la Sierra Madre orientale à l'ouest et au sud de la ville - soit près de 250000 hectares - est théoriquement protégée par ce parc naturel.

Cette qualification juridique, qui semblait avoir été oubliée pendant plus de cinquante ans, fut réactivée dans les années quatre-vingt-dix sous la pression d'habitants de lotissements implantés sur les pentes de la montagne - dans le municipe de San Pedro Garza Garcia - mobilisés contre de nouveaux projets de lotissement et pour la préservation de leur cadre de vie privilégié. Ces associations, qui se proclament écologistes, interpellent la société locale par des actions spectaculaires: blocage des rues, pétitions, manifestations pour protester contre de nouveaux projets de lotissements sur les pentes de la Sierra [Zuñiga, 1995]. Dans cette lutte pour leur cadre de vie, les associations multiplient les recours juridiques contre les autorisations délivrées par le gouvernement de l'État en contradiction avec les plans locaux d'urbanisme et le périmètre du Parc.

Malgré les oppositions très fortes des autorités locales et de l'État, comme des représentants des promoteurs immobiliers qui dénient toute existence juridique au Parc, le Parc naturel est réactivé. Cette qualification juridique fédérale autorise l'intervention des organismes fédéraux dans les conflits concernant l'urbanisation sur les pentes de la Sierra. Toutefois, les représentants de la fédération ne disposent pas de réels moyens d'inspection sur l'ensemble de la zone. C'est une procédure de "dénonciation populaire ", permettant à tous les citoyens mexicains de signaler une violation de la législation, qui rend possible l'intervention des autorités fédérales pour bloquer des travaux d'urbanisation sur les pentes de la Sierra sur la base de la réglementation du Parc national naturel. Cette procédure, amplement utilisée par les représentants des associations, oblige les autorités fédérales à réaliser une enquête pour établir les conditions de légalité des projets mis en cause.

Pour sortir de cette situation de conflits entre niveaux d'intervention sur le territoire, les autorités fédérales ont impulsé une nouvelle délimitation du Parc national en acceptant de réduire sa superficie contre l'assurance d'un contrôle plus

5 La législation fédérale introduit un partage des tâches entre les trois niveaux d'organisation administrative, la fédération reste responsable de l'autorisation et du contrôle des activités dangereuses, le gouvernement de l'État de la plus grande partie des entreprises industrielles, et les municipalités du reste des activités (commerces, restaurants, ateliers artisanaux...).

6 Lazaro Cardenas est le président de la République (1934-1940) qui a mis en place l'institutionnalisation du régime national-populaire issu de la révolution mexicaine. 
efficace de l'urbanisation par les autorités locales et de la mise en place d'un réseau d'espaces protégés par le gouvernement de l'État.

Tout se passe comme si, dans le contexte particulier de forte pression des promoteurs et des propriétaires des terrains qui représentent les familles les plus puissantes du pays, seul le poids des autorités fédérales pouvait permettre de contrôler l'expansion de l'urbanisation. Dans une autre partie de la ville, sur la pente du Cerro de la Silla, on observe un processus similaire. Un décret fédéral de monument naturel a été arraché en 1991 aux autorités fédérales par des associations protestant contre les projets de lotissements au-dessus de la limite fixée par le plan directeur.

Au sein du gouvernement de l'État, la construction de l'environnement comme champ de l'action publique a pris la forme de la création d'une administration de l'environnement, un sous-ministère de l'Écologie au sein du ministère du Développement urbain et des Travaux publics. Malgré cette proximité institutionnelle entre la nouvelle administration de l'environnement et les responsables de la planification urbaine, l'intégration des préoccupations environnementales au sein des pratiques des acteurs de l'aménagement urbain apparaît faible. Les responsables de l'écologie au sein du gouvernement de l'État prennent en charge essentiellement le contrôle des pollutions et la protection des espaces naturels. Les aspects environnementaux de la planification urbaine constituent un champ périphérique à la fois pour les responsables de l'aménagement urbain et pour ceux de la protection de l'environnement.

Au Mexique, les modalités de contrôle de l'urbanisation relèvent à la fois de l'ordre juridique de l'urbanisme et de celui de l'environnement. À Monterrey, un intense débat relayé par les médias et les partis politiques locaux a précédé l'approbation en mars 1999 d'une nouvelle loi de développement urbain de l'État du Nuevo León. Les représentants de l'Association des promoteurs immobiliers (Adivac), qui conservent un poids important au sein du Conseil consultatif du développement urbain (Codeur), ont tenté de poursuivre leur stratégie de déréglementation. En 1997, ils avaient réussi à impulser une initiative de loi avortée qui supprimait l'ensemble des documents de planification urbaine, permettait de classer tous les espaces libres proches de la ville en terrains à urbaniser et obligeait les autorités publiques à introduire gratuitement les infrastructures [Garcia Ortega, 1998].

Conformément à la législation nationale, la loi de développement urbain adoptée en 1999 a attribué aux municipalités le contrôle direct de l'urbanisation. En outre, elle intègre deux propositions des promoteurs immobiliers : la réduction de la taille minimum d'un lot constructible et la possibilité de fragmenter les superficies de dotations municipales. Ces nouvelles concessions aux promoteurs ${ }^{7}$ se traduiront vraisemblablement par une réduction de la qualité de vie dans les nouveaux lotissements populaires.

À l'opposé, les discussions sur l'adoption d'une nouvelle loi de protection de l'environnement - qui n'ont pu aboutir - sont restées dans le cadre des processus

7 En 1993, la précédente réforme avait déjà réduit la superficie de dotation municipale et la taille minimale des lots. 
d'enquête publique et des débats entre spécialistes de l'environnement et associations écologistes. Abandonnant le champ urbain, le principal enjeu de cette loi semble être de renforcer l'efficacité et la cohérence des actions fédérales, de l'État et des municipalités dans le domaine de la protection des espaces naturels.

L'existence de deux secteurs de l'administration publique semble se traduire par la marginalisation des acteurs de la protection de l'environnement, exclus des débats sur la loi de développement urbain et, dont les propositions sont renvoyées à la loi environnementale. Or les débats sur la loi environnementale se limitent à la revendication d'un renforcement de l'administration environnementale (transformation du sous-ministère de l'Écologie en ministère, renforcement de la participation populaire, droit à l'information, création d'un Ombudsman environnemental).

Depuis une dizaine d'années, les acteurs municipaux de protection de l'environnement ont consolidé leur présence institutionnelle. Dans les municipalités de l'aire métropolitaine de Monterrey, l'administration environnementale est prise en charge par des directions de l'écologie intégrées au sein de directions du développement urbain et de l'écologie. Les administrations environnementales locales sont plus récentes, moins riches et disposent de moins de personnels que les administrations chargées de l'urbanisme ${ }^{8}$. Peu à peu, s'est mise en place une répartition des tâches entre les responsables de l'aménagement urbain et ceux de la protection de l'environnement, qui peut être considérée comme une définition opérationnelle du champ de l'environnement.

Les directions de développement urbain ont abdiqué tout discours sur les impacts de l'urbanisation sur le milieu. Les plaintes des habitants, gênes occasionnées par les mauvaises odeurs des restaurants, la pollution des petits ateliers dispersés dans le tissu urbain, les bruits de voisinage ou le trafic automobile, sont qualifiées «d'environnementales ». Alors que l'autorisation de ces activités reste du domaine des directions de l'urbanisme, toute atteinte à la qualité de la vie est considérée comme une forme de pollution urbaine.

Certaines questions qui pourraient être considérées comme " environnementales » mais qui ne relèvent pas de la gestion quotidienne des directions d'écologie sont redéfinies comme "urbanisme ». Alors que le nettoyage des espaces publics est du domaine des directions de l'environnement, celui des lots vides - nombreux en l'absence d'une obligation de construire liée à l'achat d'un terrain dans un lotissement - sur lesquels prolifèrent les décharges clandestines reste du domaine des directions du développement urbain ou de la police administrative.

Le travail des directions d'écologie est centré sur la prise en compte des plaintes et dénonciations, la gestion des conflits d'usage du sol, le contrôle de la contamination et les impacts environnementaux, la protection des espaces verts de l'image urbaine (règlements de publicité) et l'éducation environnementale. La gestion des espaces verts semble être la principale fonction des directions d'écologie. Dans un milieu marqué par une grande sécheresse - à l'exception des pentes

8 Les directions de l'écologie disposent d'un personnel en moyenne inférieur de moitié au personnel des directions municipales de développement urbain. 
des montagnes -, le verdissement n'est pas forcément synonyme de protection des espaces naturels. Les espaces non urbanisés ne sont pas des espaces verts. Certaines formes d'urbanisation contribuant au verdissement - en particulier les lotissements paysagers de luxe - peuvent être dotées par leurs promoteurs et certains directeurs municipaux de l'écologie d'une valeur d'amélioration du cadre de vie.

Devant le manque relatif d'espaces verts, les jardins des propriétés privées apparaissent comme un patrimoine que les municipalités tentent de protéger. Assimilant leur rôle à celui de tous les arbres de la ville - le maintien du couvert végétal à l'extérieur des zones urbanisées étant de la compétence de la fédération -, les directions de l'écologie se mobilisent pour renforcer la base juridique de leur intervention à l'intérieur des jardins privés.

Les risques liés à l'imbrication entre activités industrielles et zones résidentielles - théoriquement en dehors des compétences municipales - mobilisent aussi les directions de l'écologie. La municipalité constitue la première instance vers laquelle les populations se tournent en cas d'accident ou de pollution avérée. Les municipalités se saisissent des questions affectant la santé des populations. Ces thèmes politiquement très sensibles se traduisent souvent par des déclarations d'hommes politiques locaux se portant garants de «droits fondamentaux » au cadre de vie et à la santé et remettant en cause le fonctionnement des administrations environnementales de l'État et de la fédération responsables de la protection contre les risques industriels.

À l'exception de San Pedro Garza Garcia, toutes les municipalités de la zone métropolitaine ont publié des règlements d'écologie qui délimitent leur champ d'action et les procédures administratives à mettre en œuvre. Dans ce municipe où sont localisés les meilleurs lotissements de la ville et les sièges de grandes entreprises locales, toute tentative de publier un règlement d'écologie est considérée comme susceptible de déclencher une polémique sur la légitimité du contrôle municipal de la croissance urbaine. Les instances municipales sont ici soumises à deux pressions contradictoires: la conflictualité des propriétaires de terrains et promoteurs qui peuvent mobiliser d'importantes ressources juridiques contre toute action considérée comme une limitation excessive des droits liés à la propriété; et les mobilisations des habitants pour la protection de leur cadre de vie et de la "nature », qui obligent la municipalité à une vigilance renforcée. Paradoxalement, la gestion de la direction de l'écologie de San Pedro est considérée comme un modèle par les spécialistes locaux de l'environnement. En l'absence d'un règlement d'écologie, l'action publique environnementale locale repose sur le règlement du Plan directeur urbain. Dans ce municipe, les documents d'urbanisme doivent faire la preuve de leur validité juridique pour s'imposer à la population.

\section{Une recomposition de l'aménagement urbain?}

À Monterrey, l'intégration des préoccupations « environnementales » au sein des documents d'aménagement urbain a d'abord pris la forme de tentatives pour fixer une limite à l'urbanisation et protéger les espaces naturels proches. Sous la pression d'habitants mobilisés pour la protection de leur cadre de vie, la réactivation au début des années quatre-vingt-dix, puis la nouvelle délimitation en 2000 
du Parc national ainsi que le décret de monument national du Cerro de la Silla en 1991 ont permis de protéger de la pression de l'urbanisation les pentes des montagnes qui ferment l'horizon de la ville. Si, depuis 1988, la planification urbaine à l'échelle de l'agglomération avait intégré une vaste zone de protection écologique autour de la ville, celle-ci, qui n'interdit pas l'urbanisation mais la conditionne à des mesures de compensation des impacts environnementaux, est aujourd'hui en grande partie occupée par des industries, zones commerciales et lotissements paysagers. Cette zone, représentée sous la forme d'une ceinture verte autour de la ville sur les plans affichés aux murs des bureaux de tous les responsables administratifs de la ville, permet de manifester la bonne volonté environnementale des praticiens de l'aménagement urbain.

La nouvelle délimitation du Parc national et la zone de protection écologique s'attachent à fixer des limites à l'urbanisation, à introduire une référence à la protection de la nature dans les documents d'urbanisme et les pratiques des acteurs de l'aménagement urbain. L'invocation du paradigme environnemental est plus nettement présente dans la mise en œuvre locale de procédures nationales d'aménagement écologique du territoire et dans la publication de nouveaux documents d'aménagement métropolitain présentés comme une refonte de la gestion urbaine à partir de la nécessité de contrôle de la qualité de l'air.

En déterminant des « vocations » de l'espace non urbanisé, le processus d'aménagement écologique du territoire doit servir de support pour déterminer les usages du sol et la localisation optimale des différents types d'activités économiques. Ce nouveau champ de l'aménagement a été créé dès les années quatrevingt mais son exercice ne se généralise au niveau local qu'à la fin des années quatre-vingt-dix. Dans un contexte de perte de vitesse de l'aménagement du territoire, de développement d'une rhétorique de planification stratégique, il caractérise un retour d'une tentative de planification spatiale intégrant les plans nationaux, régionaux et locaux. D'ailleurs, pendant le mandat du président Ernesto Zedillo (1994-2000), les tentatives de renforcer le rôle institutionnel de la planification écologique ont été prises en charge par des spécialistes qui avaient joué un rôle important dans l'essor de l'aménagement du territoire dans les années soixante-dix ${ }^{9}$. Dans des situations fragiles ou conflictuelles et dans certaines municipalités rurales, cette procédure a permis d'identifier les milieux à protéger. Mais dans le domaine urbain et régional, les documents d'aménagement écologique du territoire sont restés, le plus souvent, des exercices techniques sans prise sur les modalités de contrôle de l'urbanisation [INE, 1999].

Néanmoins, la protection de l'environnement a été intégrée aux discours des documents de planification urbaine. Le nouveau plan de développement urbain de l'État du Nuevo León, publié en 1999, sanctionne l'existence d'un autre champ de la planification. Le chapitre "écologie et environnement " programme la formulation d'un document d'aménagement écologique du territoire à réaliser au niveau de l'État mais aussi de chacun des municipes, dans le but de fixer des limites stables à l'urbanisation.

9 Au sein de Sahop, ministère des Établissements humains et des Travaux publics (1976-1982). 
La municipalité de Monterrey a réalisé le seul exercice de planification écologique mené à son terme dans l'agglomération. Le plan d'aménagement écologique de Monterrey-sud, publié en 1995, traite du Cañon de Huajuco, espace situé au sud-ouest de la municipalité, entre la Sierra Madre et le Cerro de la Silla. En partie protégé par le Parc naturel, le Cañon de Huajuco est occupé par des lotissements populaires et « campagnards ${ }^{10}$ », pour certains illégaux. La municipalité de Monterrey envisage une urbanisation contrôlée, seule façon d'introduire les infrastructures permettant d'assainir les lotissements existants et ainsi de protéger le milieu.

Le zonage proposé par le plan écologique organise la construction de lotissements campagnards paysagers et la réalisation de petits parcs écologiques par acquisition foncière. L'intégration du Cañon du Huajuco au développement urbain contrôlé du municipe est présentée comme la seule façon de protéger non seulement le milieu mais aussi le paysage des pentes de la montagne. Dans cette optique, les parcs écologiques et les lotissements paysagers composés de luxueuses villas entourées de vastes jardins constituent une nouvelle forme d'urbanisation «préservant» certains éléments du milieu (respect des pentes et des écoulements, maintien d'un grand nombre d'arbres) et assurant aux acheteurs une position privilégiée et la stabilité de leur proximité de la nature. Cet exercice de planification écologique, dont l'efficacité est à analyser dans le cadre des négociations entre la municipalité et la fédération sur les nouvelles limites du Parc national, propose une vision d'un " aménagement urbain écologique ", offrant des garanties de gestion du milieu en échange d'une ouverture à l'urbanisation et d'un renforcement de la division sociale de l'espace urbain. L'implantation de lotissements populaires incontrôlés est perçue comme le principal risque pour les espaces naturels proches de la ville ; ceux-ci sont rejetés sur les espaces arides peu attractifs pour les classes moyennes ou aisées.

La publication d'un Programme d'administration de la qualité de l'air de la zone métropolitaine de Monterrey (1997-2000) semblait incarner la volonté de renforcer la planification urbaine à partir de la mise en œuvre de mesures de contrôle de la pollution. Une série d'actions concertées entre les neuf municipes de la zone métropolitaine était proposée, intégrant aménagement urbain et environnement.

La mise en cohérence des politiques d'infrastructures, de transports, d'attribution des autorisations de construire, de gestion des réseaux et services urbains, est censée modifier les modalités de la croissance urbaine. Il s'agit de produire une agglomération dont la forme rend possible la diminution de la circulation automobile et donc de la pollution de l'air. Assez classiquement, la réduction des émissions est attendue de la production d'une ville plus compacte et de l'amélioration du réseau de transports en commun (création de nouvelles lignes d'autobus et extension du métro).

Malgré l'invocation de la nécessaire mobilisation de l'ensemble des acteurs de l'aire métropolitaine, l'élaboration de ce texte n'a pas ouvert un espace local de

10 Les lotissements campagnards (campestre) constituent une catégorie du droit de l'urbanisme permettant de lotir pour la réalisation de résidences secondaires entourées de vastes jardins. 
débat sur les relations entre aménagement urbain et protection de l'environnement. Comme dans les exercices de planification du même type réalisés à Mexico [Lezama, 1997] et Guadalajara, les représentants et les consultants de la Banque mondiale ont joué un rôle déterminant. Le diagnostic, les modalités d'analyse et les mesures proposées sont similaires dans les trois villes. Toutefois, si les études ont été financées par la Banque mondiale, les ressources nécessaires à la réalisation des actions listées dans ce programme n'ont pu être débloquées. Malgré la volonté affichée d'impulser une mutation des formes de la croissance urbaine, les effets ont été beaucoup plus limités. Seuls ont pu être mis en place des actions de formation pour les responsables municipaux de l'environnement et, pendant un temps, un programme de contrôle technique des véhicules usagés intitulé « ciel propre ".

Au total, le renforcement de la protection de l'environnement apparaît comme une question mobilisant d'abord les institutions fédérales. À Monterrey, pour assurer la stabilité de leur environnement proche, les habitants mobilisés dans des conflits urbains pour la protection de la nature demandent l'intervention de la fédération. Les documents d'urbanisme et les procédures locales de protection des espaces naturels sont jugés insuffisants pour s'imposer aux propriétaires des terrains et aux promoteurs. Les administrations fédérales apparaissent comme les seules autorités suffisamment dégagées des enjeux locaux pour garantir la validité des mesures de contrôle de l'urbanisation et de protection des espaces naturels qui entourent la ville. Celles-ci, dans l'incapacité d'assurer une présence sur le terrain, s'appuient sur la vigilance des populations ou sur des organes de gestion des espaces protégés intégrant la participation de la population.

L'environnement constitue un nouveau secteur de l'action publique locale, construit d'une manière parallèle et séparée du champ de l'aménagement urbain. L'action publique environnementale est dans l'incapacité non seulement de se saisir du contrôle de l'urbanisation, mais aussi d'introduire un débat sur les responsabilités «environnementales» des modalités de production de l'espace urbain. L'impératif environnemental n'a pas modifié la façon de mettre en œuvre l'aménagement urbain. Une action publique environnementale encadrée par un ordre juridique spécifique et des institutions spécialisées a néanmoins été mise en place à l'échelle locale, concernant la gestion des infrastructures et des services techniques, les espaces verts et la police des pollutions. Mais les administrations de l'environnement ont ouvert un nouveau canal d'expression des conflits urbains.

À Monterrey, les contraintes imposées à la promotion immobilière par la législation urbaine ont été réduites, ce qui permet la construction de nouveaux espaces urbains plus denses et potentiellement marqués par des conflits d'usages et de voisinage. Or, dans le même temps, s'est généralisée une action publique environnementale au niveau municipal qui tente de limiter les pollutions et les atteintes à la qualité de vie des habitants. Incapables de peser sur les modalités de la croissance urbaine ou sur le contrôle de la localisation des activités, les directions d'écologie concentrent leurs actions sur la négociation de mesures techniques de réduction de troubles de voisinage ou des atteintes à la qualité de la vie. 
La société locale est marquée par le poids d'une puissante bourgeoisie industrielle dont les membres contrôlent aujourd'hui tous les rouages des pouvoirs locaux. Le renforcement de l'efficacité de l'action publique urbaine et environnementale est assimilé à une imposition et une limitation à leur capacité d'initiative. Les directions de l'environnement semblent constituer un écran entre les demandes des populations et les responsables du contrôle de l'urbanisation. Porteurs de références au bien commun exprimées dans le langage du paradigme environnemental, les responsables de l'environnement ne peuvent être soupçonnés de collusion avec les intérêts du développement urbain ou industriel. Les directions municipales de l'écologie apparaissent comme des instances de canalisation des plaintes et des conflits urbains mettant en œuvre une reconnaissance symbolique du droit à « l'environnement » des populations.

Le paradigme environnemental et les références à des causalités systémiques appelant une mutation des pratiques sociales reviennent à évacuer le débat sur le rôle des procédures de contrôle de l'urbanisation. On peut considérer que les actions d'éducation environnementale n'ont pas pour seul rôle de construire le champ de l'environnement, en présentant les liens systémiques entre la gestion des déchets familiaux et la pollution industrielle, le verdissement de la ville et la protection de la nature; l'efficacité de ces actions semble être aussi de diluer la responsabilité de certaines formes de production de l'espace urbain dans une responsabilité globale qui implique les pratiques quotidiennes de chaque habitant.

Instituant le paradigme environnemental mais construite à partir de pratiques ponctuelles fragmentées, l'action publique environnementale est soumise à l'injonction de faire la preuve de sa cohérence. L'évaluation des réductions des atteintes au milieu et à la qualité de la vie des populations n'importe pas tant que la démonstration du respect des principes et modèles d'actions publiques légitimes, constituant la doxa environnementale. Mutations législatives, innovations institutionnelles et programmes locaux fonctionnent - indépendamment de leur consistance et validité - comme signes de la mise en œuvre d'une action environnementale conforme à cette opinion globale relayée par de nombreux acteurs locaux. En ce sens, la construction de l'action publique environnementale à Monterrey constitue une modalité d'existence locale d'un paradigme et de modèles d'action publique construits globalement, intégrés au sein de l'ordre juridique et des procédures administratives nationales, mais aussi directement présents dans les relations entre acteurs locaux comme principe de jugement et de justification de l'action [Lafaye, Thévenot, 1993].

\section{BIBLIOGRAPHIE}

Atrinson A., Davila J., Fernández E., Mattingly M. (eds) [1999], The Challenge of Environmental Management in Urban Areas, Hants, Ashgate Publishing Company, 298 p.

Bañuelos M. (coord.) [2000], Sociedad, derecho y medio ambiente, México, Profepa, Semarnap, Conacyt, UAM, $541 \mathrm{p}$.

Bassols M., Melé P. (coord.) [2001], Medio ambiente, ciudad y orden jurídico, México, Miguel Angel Porrua, UAM, 420 p.

Camagni R., Gibelli M.-C. (dir.) [1997], Développement urbain durable, quatre métropoles européennes, L’Aube, 174 p. 
FarThing S.M. (ed.) [1997], Evaluating Local Environmental Policy, Hants, Ashgate Publishing Company, $191 \mathrm{p}$.

FERnANDes E. (ed.) [1998], Environmental Strategies for Sustainable Development in Urban Areas, Lessons from Africa and Latin America, Hants, Ashgate Publishing Company, 212 p.

García OrTEga R. [1998], Área metropolitana de Monterrey 1985-1997, El Colegio de la Frontera norte.

GonZALEZ Marquez J.J. [1997], Nuevo derecho ambiental mexicano (instrumentos de política), México, UAM, Azcatpozalco, 353 p.

INE [1999], Primer congreso nacional de ordenamiento ecológico, hacia una nueva planeación del territorio de México siglo $\mathrm{XXI}^{e}$, Consejos consultativos para el desarrollo sustentable, Instituto Nacional de Ecología, Pnud, Guadalajara.

Kürzinger F.E. et alii [1991], Política ambiental en México, el papel de las organizaciones no gubernamentales, México, Instituto Alemán de Desarrollo.

Lafaye C., Thévenot L. [1993], « Une justification écologique? Conflits urbains pour la protection de la nature », Reoue française de sociologie, XXXIV : 495-524.

Lascoumes P. [1994], L'Éco-pouvoir, environnement et politique, Paris, La Découverte, 317 p.

LEZAMA J.L. [1997], « La construcción gubernamental de la contaminación ambiental: la política del aire para la ciudad de México 1979-1996 », México, economía, sociedad y territorio, 1 (2), juillet-décembre 1997, El Colegio Mexiquense, 317-364.

Marvin S., GuY S. [1999], "Beyond the Myths of the New Environmental Localism ", in The Challenge of Environmental Management in Urban Areas, Hants, Ashgate Publishing Company: 203-213.

MeLÉ P. [2001], « Pouvoirs locaux et recompositions de l'action publique urbaine au Mexique », in Les Territoires de l'État-nation en Amérique latine, Paris, Éditions de l'IHEAL: 47-63.

Melé P., Bassols M. (coord.) [2000], Environnement, Ordre juridique et Gestion urbaine, México, VST, université de Tours, UAM Iztalapalapa, rapport final pour le Programme de recherche sur l'application et l'évaluation de la législation environnementale, Profepa/Conacyt, $341 \mathrm{p}$.

Micoud A., Valarié P., Franguiadakis S. [1996], L'Écologie urbaine au risque de la cité, SaintÉtienne, Crésal-CNRS.

RudolF F. [1998], L'Environnement, une construction sociale, pratiques et discours sur l'environnement en Allemagne et en France, Strasbourg, Presses universitaires de Strasbourg, 184 p.

ZuñIga V. et alii [1995], Democracia cotidiana y sociedad en Monterrey y San Pedro Garza Garcia, Reporte de investigación, Fundación Ford, El Colegio de la Frontera Norte. 